\title{
Manufacture of 3D Curved Profiles for Structure Components
}

\author{
D. Becker ${ }^{1, a}$, M. Schikorra ${ }^{1, b}$ and A. E. Tekkaya ${ }^{1, c}$ \\ ${ }^{1}$ Institute of Forming Technology and Lightweight Construction, \\ Technische Universität Dortmund, Baroper Str. 301, 44227 Dortmund, Germany \\ adirk.becker@iul.uni-dortmund.de, bmarco.schikorra@iul.uni-dortmund.de, \\ cerman.tekkaya@iul.uni-dortmund.de
}

Keywords: Extrusion, curved profile, material flow, lightweight construction.

\begin{abstract}
Curved Profile Extrusion (CPE) is an extension of the common extrusion process and offers the possibility to manufacture three-dimensionally curved profiles. Due to the flexibility of the process different curvatures can be produced with the same setup, which makes this technique efficient especially for low volume production. The process is characterized by a controlled lateral deflection of the strand which influences the material flow in the die and causes the profile curvature. In this paper, a direct comparison of the power rating between warm bending and CPE is presented. Furthermore, the investigations concerning the choice of support strategy of the surmounting profile by a robot to increase the curvature accuracy are continued. Finally, some extensions of the equipment are explained to increase the level of process integration.
\end{abstract}

\section{Introduction}

Modern transportation engineering applies multiple types of lightweight construction, concerning e.g. the materials science, design, and manufacturing. In the automotive industry the use of aluminum in structure components is shown by Audi in the A8 and, furthermore, in the sports car $\mathrm{R} 8$ to reduce the weight by up to $40 \%$. The application of extruded profiles in these space frames offers different designs of cross sections so that an optimization by topology can be accomplished. Due to the wide choice of cross sections an integration of functions can be obtained, which supports the reduction of weight [1,2]. Thinking further in this direction, the large number of joints could be reduced using more curved profiles. In general, curved profiles are obtained by a bending process which influences unfavorable the profile geometry by elastic springback, cross section deformation, and the reduced formability. In contrast to this, the innovative process Curved Profile Extrusion (CPE), which was developed by the Institute of Forming Technology and Lightweight Construction, avoids these negative properties in workpieces like structure components.

Curved profile extrusion is an alternative production method to the conventional process chain to manufacture curved profiles. This production type is based on the conventional bar extrusion process in which the exiting strand is deflected by a numerically controlled guiding tool so that the curvature is based on a kinematic shape generation. On the one hand, the deflection of the profile causes compression stress at the inner bearing and amplifies the friction. On the other hand, tensile stress is superposed at the outer bearing which increases the material velocity. Due to the already plasticized material in the die the superposed moment produces no springback, and no residual stress in the curved profile. Additionally, the material of the manufactured profile offers nearly the full formability so that a following forming process, e.g. hydroforming, is still possible to add further details in design $[3,4,5]$. 


\section{Process Comparison}

A more detailed analysis of CPE helpful to separate this warm forming process from a conventional warm bending process especially regarding the sensitivity against external influences is obtained by the comparison of the loads which are required to achieve the curvature. Both process experiments are applied directly at the extrusion press with the same profile geometry of a round rod of $36.5 \mathrm{~mm}$ diameter to establish comparable initial conditions. The extrusion speed is set to $2 \mathrm{~mm} / \mathrm{s}$ for the ram corresponding to $32 \mathrm{~mm} / \mathrm{s}$ for the profile speed. This leads to an exiting temperature of the profile between $430^{\circ} \mathrm{C}$ and $450^{\circ} \mathrm{C}$, using a preheated billet at $550^{\circ} \mathrm{C}$ and a container temperature of $450^{\circ} \mathrm{C}$. The linear axis system which is installed for curved profile extrusion is also used for the warm bending process. In order to record the force of the deflection, a load cell is integrated in the guiding tool system, displayed in fig 1 .

a)

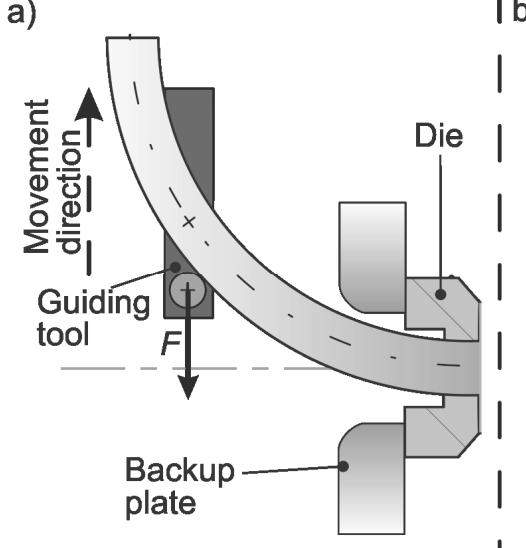

lb)

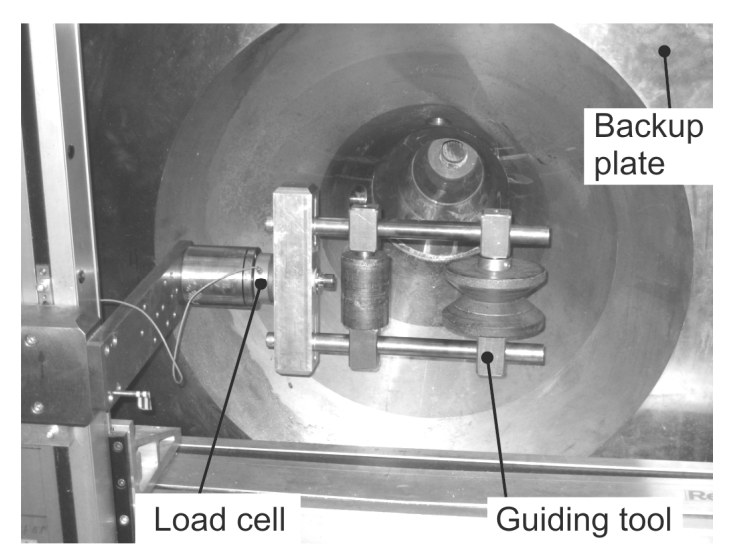

Fig. 1: a) Sketch of the force measurement direction; b) Real setup at the press

In general, the guiding tool for CPE has two rotatory degrees of freedom so that it orientates automatically in tangential direction to the profile. But for the force measurement this rotatory movements should be prevented to detect the force for deflection. The deflection movement of the guiding tool was generated as described in the chapter Flexible Profile Support below and is driven by a PLC control. The CPE experiment is applied by a synchronous movement of the guiding tool to the profile speed to produce a constantly plane curved profile with a radius of 1,500 $\mathrm{mm}$. In contrast to this, the warm bending test is not operated parallel to the extrusion process. First, the extrusion process is started and run until the steady state is reached, which is the case when the target speed is attained and the press force slowly decreases. Then, the press stops and the same deflection of the guiding tool starts to introduce the bending process. The results of both force measurements are shown in fig. 2.

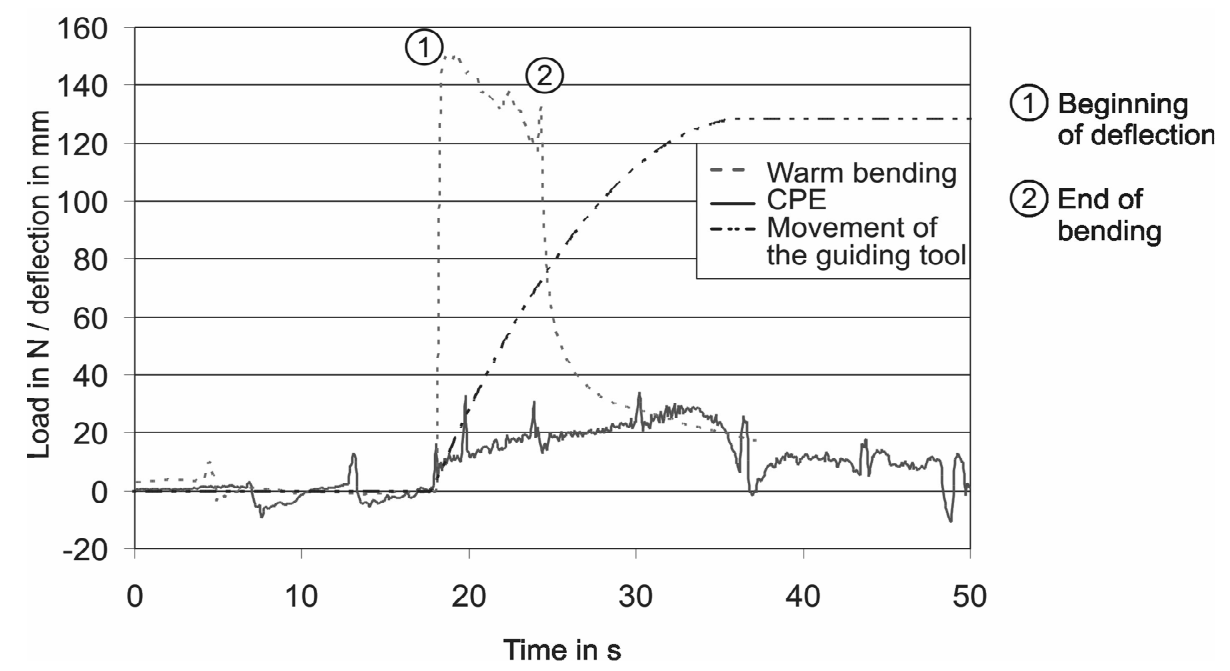

Fig. 2: Load results of warm bending and CPE 
The diagrams indicate that $\mathrm{CPE}$ requires only minimal forces of maximal $34 \mathrm{~N}$ to deflect the strand for the curvature generation. In comparison to CPE, the warm bending process needs initial forces which are nearly five times higher (up to $150 \mathrm{~N}$ ) to deflect the profile. This circumstance makes the definite difference between CPE and warm bending obvious, but also makes it necessary to move the guiding tool on the correct path without any external influencing forces which overdetermine the manufacturing process. Some of the influencing factors are discussed in the following.

\section{Compensation by Profile Support}

Factors of Influence. The accuracy of the curvature depends on several influencing factors. The detailed analysis of the geometrical condition of the manufacturing cell has to be completed to assure correct deflections corresponding to the desired profile line. As it was investigated by Klaus [4], the geometrical parameters, the distance between guiding tool and die, the distance of the deflection and the angle between the press axis and the linear axis are determined by a practical calibration of the system assisted by a non-linear optimization. Due to the warm forming process the thermal conditions in the die as well as in the profile have to be taken into account. The profile line can also be influenced by quenching after the exit of the profile so that some sections distort more than others which largely depends on the cross section design. Because of the consideration of three-dimensionally curved profiles the use of a table for the profile support in the run out of the extrusion press is not possible. Therefore, bending and torsion effects occur due to the influence of gravity [6].

Flexible Profile Support. Introduced in [6], the profile support is realized and investigated for plane curved profiles by a six-axis robot type Kuka KR150-2 with a second guiding tool attached to the robot's arm. The results of support strategies applied with a constant distance between the first and the second guiding tool for constantly curved profiles have to be transferred to the manufacture of three-dimensionally curved profiles by CPE. Due to the complex geometries of threedimensionally curved profiles the movement for the profile support behind the guiding tool has to be very flexible. The calculation of the movement data for the constantly plane curved profiles is easy, but considering the additional dimension in the curvature requires a movement path similar to a 3D-spline. Now, the support strategy is expanded from the constant distance between the first and the second guiding tool to an attendant support of the profile. By the help of a CAD/CAM chain the movement data for the complex profile shape can be generated. The process chain begins with the $\mathrm{CAD}$ model of the desired structure profile from which the neutral line has to be extracted. This line is imported into a CAM system applicable for kinematics simulation. There are two CAM systems used in the Collaborative Research Center which are CATIA V5 with the module DMU kinematics or MOSES, which is an added application to AutoCAD [7,8]. Both CAM systems offer the possibility to simulate the manufacturing process in which the profile is guided tangentially through the die. Additionally, the movements of the guiding tool and the robot can be checked regarding the working range of the kinematics and the collision with the components of the manufacturing cell before the real process takes place. During the simulation the generation of the movement data for the guiding tool and the tool center point of the robot are recorded. The program code is exported to the robot system where the real tests of the movements are made before the production begins. The described experiments are carried out with the help of the CAM system MOSES. A surface in the virtual production cell defines the support position of the second guiding tool. In this case, the surface is a hemisphere with the center point at the position of the bearing. In the beginning of the extrusion process the second guiding tool starts at a position directly behind the first guiding tool and follows the profile synchronously to the profile velocity. If the tool center point of the second guiding tool reaches the hemisphere, it only moves along the surface, but will not leave it for the rest of the process (fig. 3). 


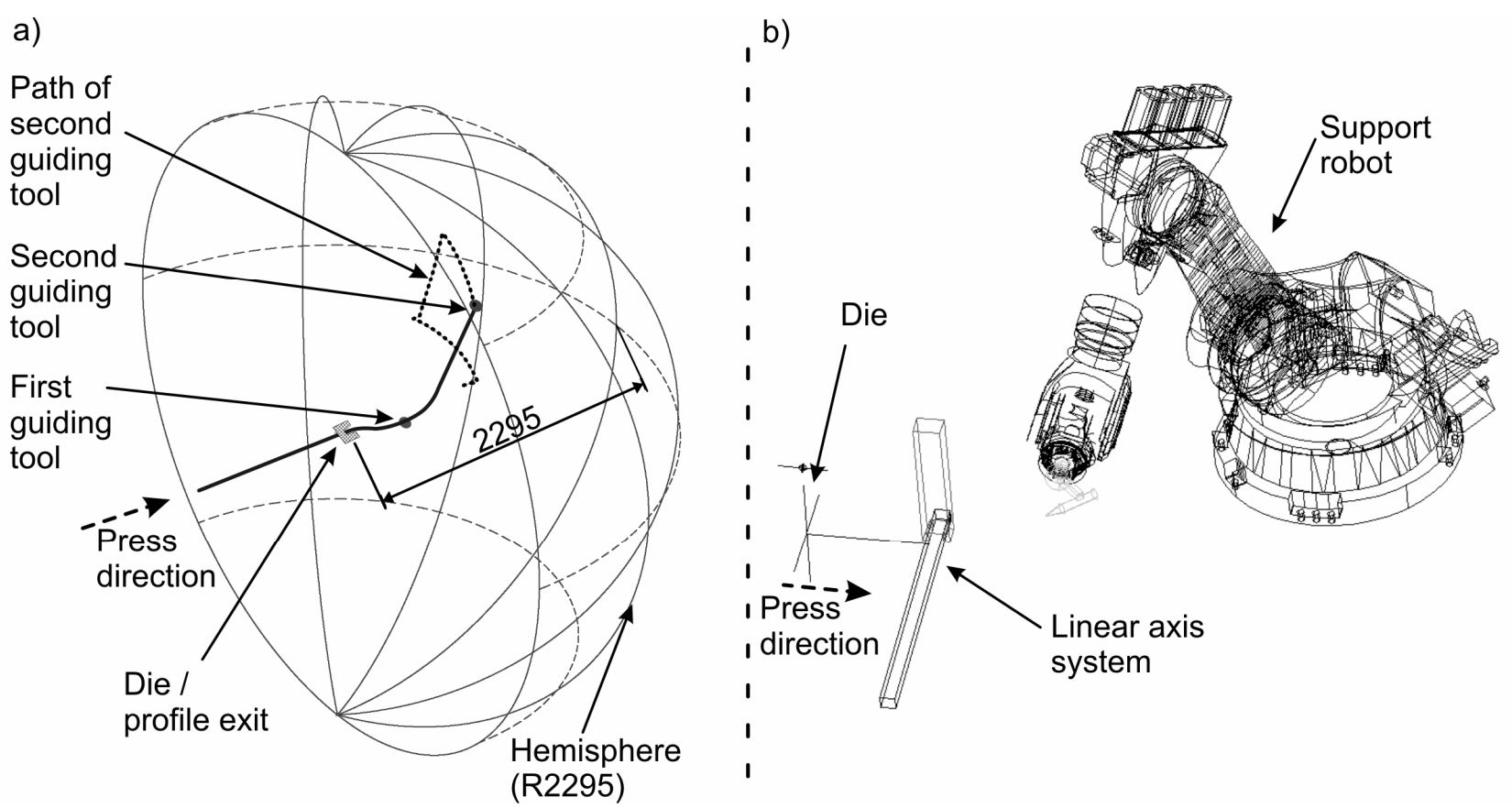

Fig. 3: Data generation cell (a) and virtual robot cell (b)

The chosen hemisphere as support surface has to be adjusted by the parameters radius and distance from the die concerning the accuracy of the manufactured profile. With regard to the bending and torsional stresses in the profile calculated by means of mechanical equations two radii of the hemisphere, $2085 \mathrm{~mm}$ and $2295 \mathrm{~mm}$, both with their center at the position of the die, were applied for one part of the demonstrator profile frame, the motorcycle BMW C1 (fig. 4).

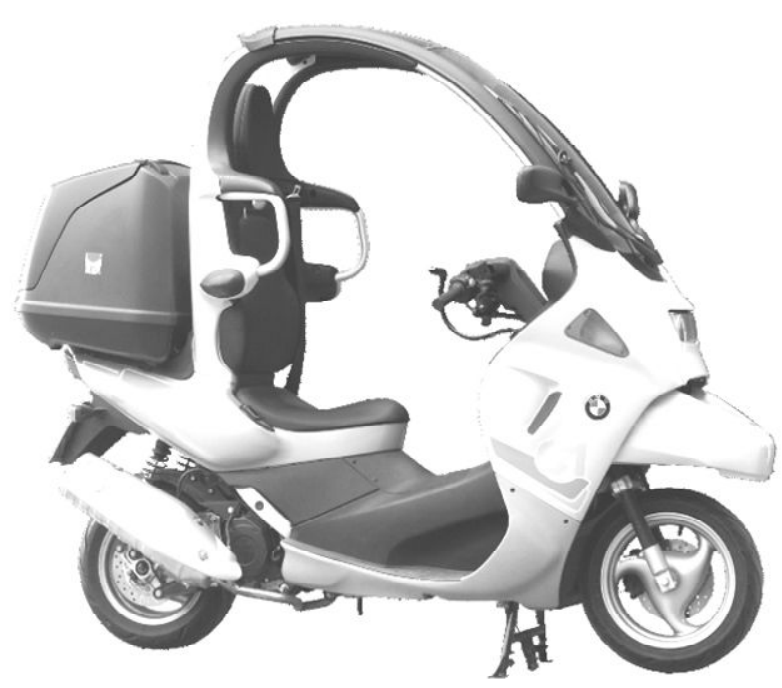

BMW C1

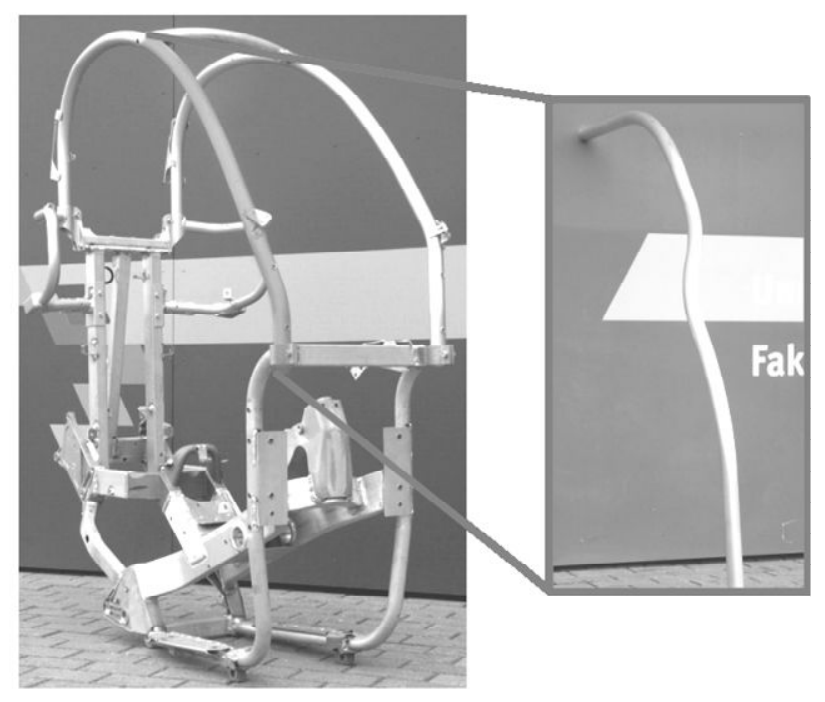

Original space frame
Structure component (roof rail)

Fig. 4: Demonstrator vehicle and structure

The results of the experimental investigations are shown by a shape comparison between the target model and the manufactured structure component with the help of a 3D optical digitizer (GOM: ATOS I+Tritop) displayed in fig. 5. Considering the entire profile for the best-fit analysis, the deviations in some very few positions on the profile reach up to $+/-7 \mathrm{~mm}$. In large parts the profile obtains the target contour with a deviation of $+/-3 \mathrm{~mm}$. 


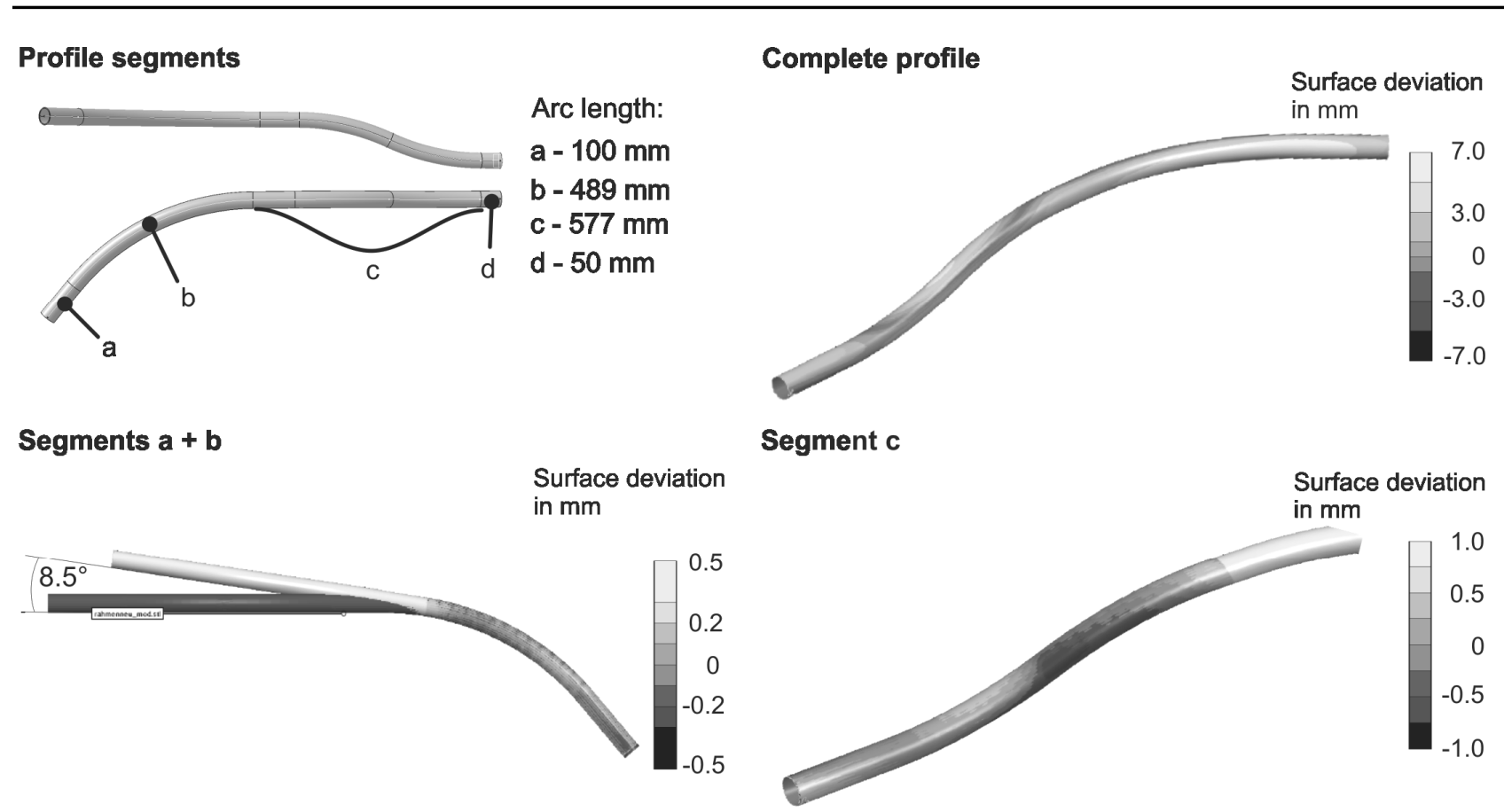

Fig. 5: Curvature accuracy of different profile segments

The profile accuracy can be examined regarding the segments of the profile, which are distinguished by the movement of the guiding tool during the manufacturing process. In fig. 5 , the four segments $\mathrm{a}, \mathrm{b}, \mathrm{c}$, and $\mathrm{d}$ with the following characteristics are displayed.
a - straight profile at the beginning
b - constantly plane curved part
c - vertical s-shaped part
d - straight profile at the end

The correct initial configuration of the manufacturing cell will be shown by the accuracy of the straightness in the segment a. All following parts depend on the accuracy of the previous parts. This is the reason why the failure will increase more and more towards the end of the profile. With respect to the front part, in particular segment $\mathrm{a}$ and $\mathrm{b}$, the deviations are determined predominantly in the arc length. The difference between the manufactured and target angle is measured with $8.5^{\circ}$. Contrary to this, the surface deviation amount to a maximum of $+/-0.5 \mathrm{~mm}$ towards the end of the arc of the manufactured profile. One reason of this deviation can be found in the performance of the synchronization between the profile speed and the deflection movement of the guiding tool. The synchronization of the presented experiments is applied by the ram speed and the press ratio, which differs due to the elongation of the press frame and the unsteady temperature conditions. The s-shaped segment most likely achieves the desired accuracy of $+/-1 \mathrm{~mm}$. Although the surmounting profile behind the second guiding tool is very long, approximately more than $1,000 \mathrm{~mm}$, the straightness of the last part is identified with $+/-0.5 \mathrm{~mm}$.

\section{Manufacturing Cell for CPE}

Extension of the Movement Range. In the Collaborative Research Center TR10 the complete process chain of forming, cutting, and joining is investigated. Therefore, one aim in this wide research work is the cooperation between the individual subprojects, which leads to many interfaces in communication and also in the real process chain. In particular, the forming process by CPE is followed by the flying cutting described in the paper "accuracy of a flying cutting device" of this 
issue, displayed [fig. 6a]. Both subprojects use a 6-axis robot which has to be synchronized and very flexible according to the curvature of the desired profile. Thus, the flexibility in positioning the robot for its profile supporting task also during the process is increased by setting it on a linear axis (Kuka KL1500). Additionally, the spectrum of support strategies and curvatures can be enhanced by the functions of the larger working range. Fig. 6a shows the fixed positioning of the cutting and supporting robot at the state of the knowledge of the first funding period in 2006. The current setup of the linear axis is parallel to the press axis to enlarge the range in one direction (see fig. 6b). In the future, the design of the short process chain between CPE and the flying cutting is planned according to the sketch in fig. $6 \mathrm{c}$ so that the collision between the robots is prevented and it is still possible to deliver the profile by the support robot to the subsequent machining or joining cell.
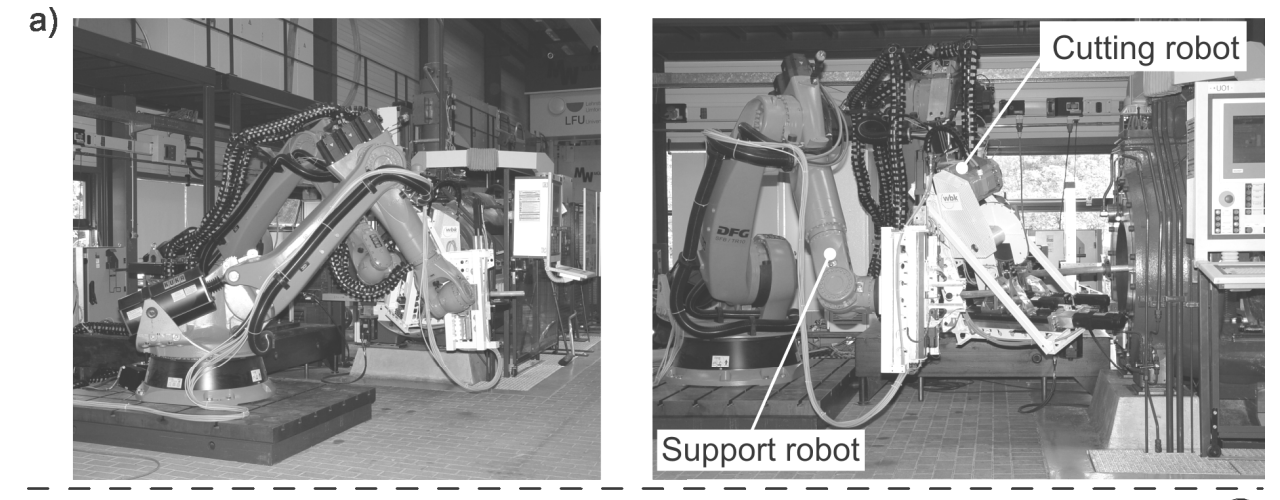

b)
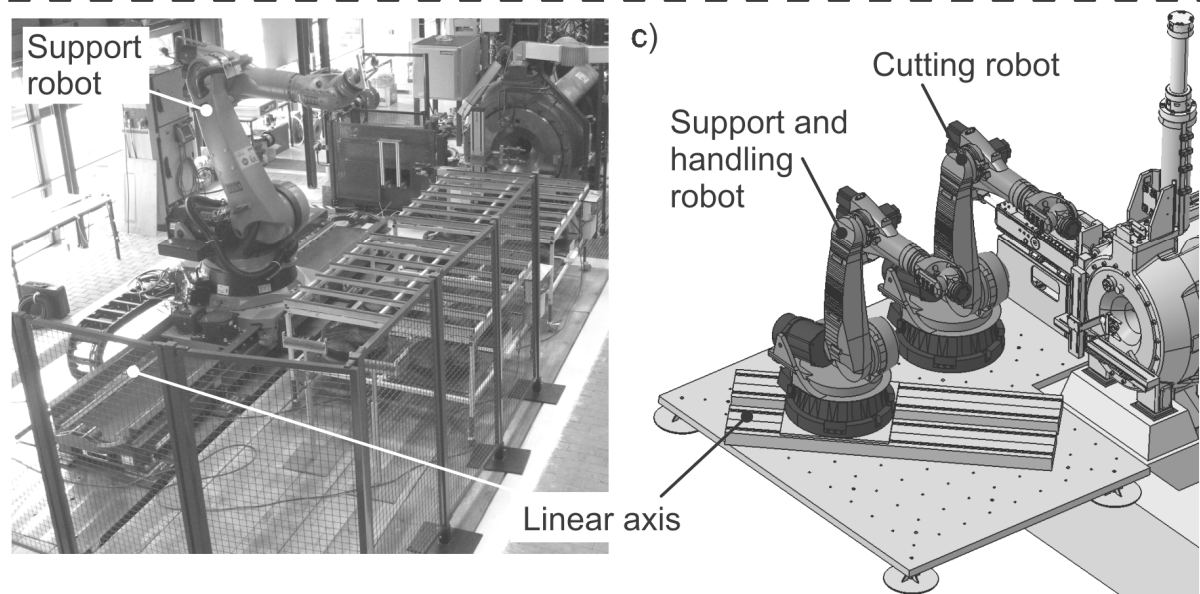

Fig. 6: Manufacturing cell: a) First setup expertise in 2006; b) Current setup;

c) Future setup with two robots

In-line Quenching. The standard aluminum alloy 6060 has to be cooled down directly after the extrusion process to preserve a fine distribution of the alloying elements. This precipitation hardening is used to achieve the required mechanical properties [9]. Due to the flexible setup of the manufacturing cell and the sideward movement of the profiles of CPE the conventional straight cooling section is not applicable. Therefore, the quenching process begins already in front of the kinematics, directly behind the die, by a simple perforated ring fed with air pressure to blow parallel towards the press direction. A second ring was installed at the guiding tool to support a fast cooling, displayed in fig. 7a. In order to minimize the sensitivity against contact with the handling kinematics later on in the process chain and to follow the objective of using high strength aluminum alloys, the cooling rate has to be increased. On this account, a spray mist quenching was developed which limits the cooling to a small area to minimize the contamination of the kinematics. In fig. $7 \mathrm{~b}$, the equipment of the spray mist cooling is displayed with four spray guns to quench the profile equally, which prevents distortion. The diagram shows the quenching effect on the profile surface cooling down from the solution temperature to below $200^{\circ} \mathrm{C}$ in a very short time so that cooling rates of $20^{\circ} \mathrm{C} / \mathrm{s}$ are possible. The low mark in the temperature curve represents the end of quenching 
by mist spray with a subsequent heat flux of the neighboring areas up to a temperature of $150^{\circ} \mathrm{C}$. The first tests show very good results, but the integration into the process chain has to be proved in the future.

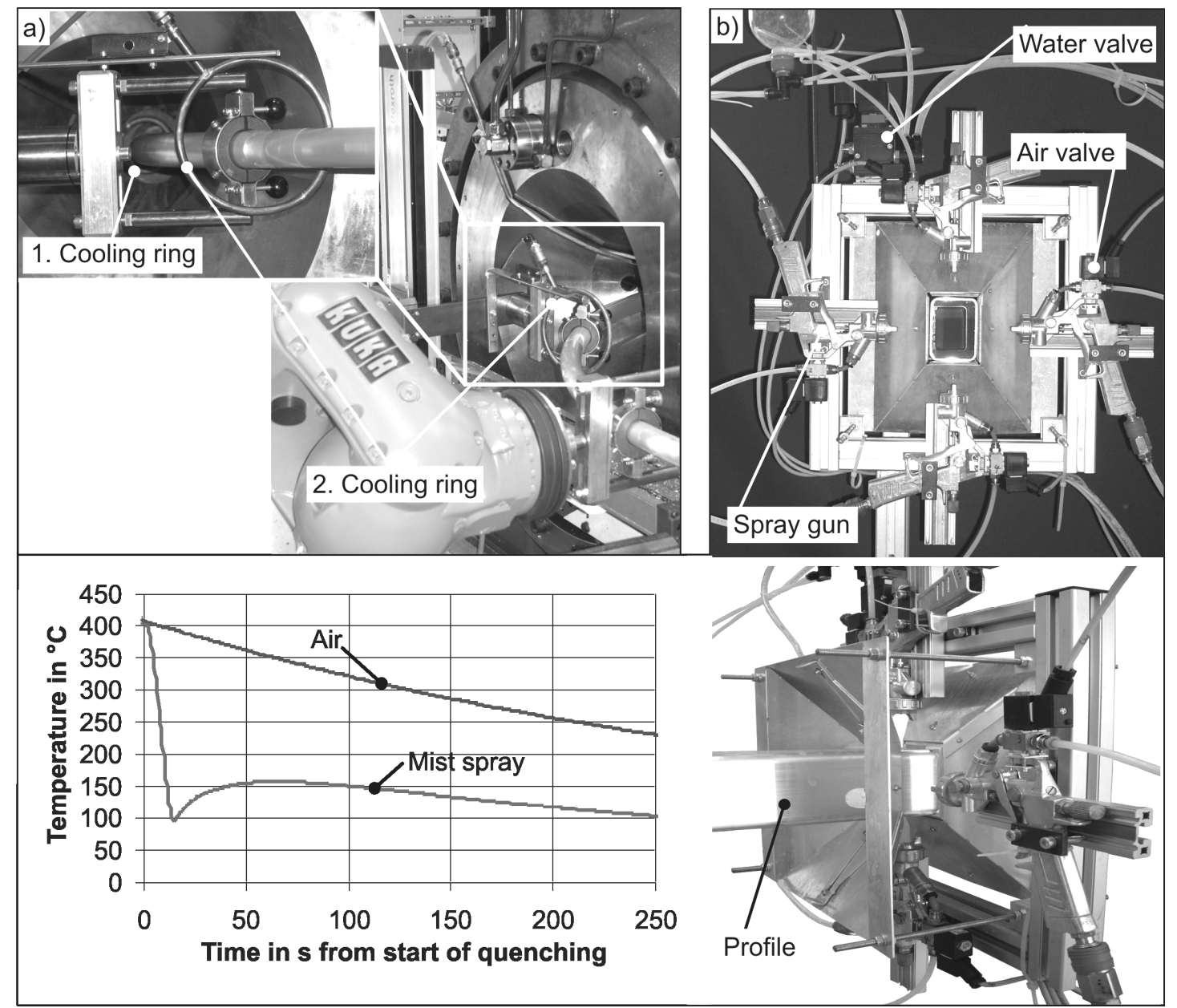

Fig. 7: a) Cooling by air pressure; b) Cooling by mist spray

\section{Summary}

The investigations show that CPE, in comparison to a conventional warm bending process, requires considerably less forming capacity so that this method is energy-efficient in addition to the better mechanical properties. Furthermore, the further development of the manufacture of threedimensionally curved profiles provides the application of complex shaped structure components. Regarding the accuracy of the profiles, the influence of gravity makes it difficult to accomplish high standards as demanded by customers like the transportation industry. The application of a robot for profile support against the effects of gravity offers a possibility to set the contour deviations to a level below $+7 /-3 \mathrm{~mm}$ and even below $+/-0.5 \mathrm{~mm}$ in some profile segments. Due to the flexibility of $\mathrm{CPE}$ and the integration in the process chain the manufacturing cell needs to be adapted in the same way. Therefore, the robot is mounted on a big linear axis to expand the working range for profile support and handling. Additionally, the quenching process is considered to implement it directly in the process chain by a fast mist spray cooling device with cooling rates of up to $20^{\circ} \mathrm{C} / \mathrm{s}$.

\section{Acknowledgment}

This paper derives from investigations within the scope of the Collaborative Research Center/TR10, which is kindly supported by the German Research Foundation (DFG). 


\section{References}

[1] H. H. Braess, U. Seiffert: Space-Frame. Chapter 6.1.2, In: Vieweg Handbuch Kraftfahrzeugtechnik, Vieweg Verlag (2007).

[2] Chr. A. Sawyer: Body Beautiful. In: Automotive Design \& Production, Gardner Publications Inc, Vol. August 2007 (http://www.autofieldguide.com/articles/080704.html).

[3] M. Kleiner: Patent EP 0706843 B1: Verfahren und Vorrichtung zur Herstellung von gekrümmten Werkstücken (1999).

[4] D. Arendes: Direkte Fertigung gerundeter Aluminiumprofile beim Strangpressen. Dr.-Ing. Dissertation (PhD-Thesis), Universität Dortmund, Shaker Verlag, Aachen 1999. ISBN 3826564421

[5] A. Klaus: Verbesserung der Fertigungsgenauigkeit und der Prozesssicherheit des Rundens beim Strangpressen. Dr.-Ing. Dissertation (PhD-Thesis), Universität Dortmund, Shaker Verlag Aachen 2002, ISBN 3-8322-0208-0.

[6] A. Klaus, D. Becker, M. Kleiner: Three-Dimensional Curved Profile Extrusion - First Results on the Influence of Gravity. In: Advanced Materials Research: Flexible Manufacture of Lightweight Frame Structures, Vol. 10 (2006), pp. 5-12, ISBN 0-87849-403-0.

[7] J. Fleischer, C. Munzinger, G. Stengel: Flying Cutting of Spatially Curved Extrusion Profiles. In: Advanced Materials Research: Flexible Manufacture of Lightweight Frame Structures, Vol. 10 (2006), pp. 5-12, ISBN 0-87849-403-0.

[8] J. Bickendorf: Automatic controller source code generation for Rounding during Extrusion. In: VDI-Berichte No. 1892 (2005) pp. 1061-1072, ISBN 3-18-091892-6.

[9] F. Ostermann: Anwendungstechnologie Aluminium. Springer Verlag 1998. ISBN 3-540-62706-5 